\title{
Association of Cigarette Smoking with Sleep Disturbance and Neurotransmitters in Cerebrospinal Fluid
}

This article was published in the following Dove Press journal: Nature and Science of Sleep

\author{
Hui Li, ${ }^{1-3}$ Yanlong Liu, ${ }^{4-6}$ \\ Lifei Xing, ${ }^{7}$ Xiaoyu Yang, ${ }^{8}$ \\ Jinzhong Xu, ${ }^{9}$ Qiushi Ren, \\ Kuan-Pin Su, iD ${ }^{4,10,11}$ \\ Yanye Lu, (D)' Fan Wang 2,3,12
}

'Department of Biomedical Engineering, College of Engineering, Peking University, Beijing 10087I, People's Republic of China; ${ }^{2}$ Xinjiang Key Laboratory of Neurological Disorder Research, The Second Affiliated Hospital, Xinjiang Medical University, Urumqi 830063, People's Republic of China; ${ }^{3}$ Psychosomatic Medicine Research Division, Inner Mongolia Medical University, Huhhot 0I0II0, People's Republic of China; ${ }^{4}$ Zhuji Institute of Biomedicine, School of Pharmaceutical Sciences, Wenzhou Medical University, Shaoxing 3 I I800, People's Republic of China; ${ }^{5}$ School of Mental Health, Wenzhou Medical University, Wenzhou, 325035 People's Republic of China; ${ }^{6}$ The Affiliated Kangning Hospital, Wenzhou Medical University, Wenzhou 325035, People's Republic of China; ${ }^{7}$ Department of Neurology, Inner Mongolia North Heavy Industries Group Corp. Ltd Hospital, Baotou 0I 4030, People's Republic of China; ${ }^{8}$ Beijing Jishuitan Hospital, Beijing 100035, People's Republic of China; ${ }^{9}$ The Affiliated Wenling Hospital of Wenzhou Medical University, Wenling 317500, People's Republic of China; ${ }^{10} \mathrm{An}$ Nan Hospital, China Medical University, Tainan, Taiwan; "College of Medicine, China Medical University, Taichung, Taiwan; ${ }^{12}$ Beijing Hui-Long-Guan Hospital, Peking University, Beijing 100096, People's Republic of China
Correspondence: Fan Wang Beijing Hui-Long-Guan Hospital, Peking University, Beijing I00096, People's Republic of China

Tel +86 I0 627I55II ext. 6429

$\mathrm{Fax}+861062716285$

Email fanwang@bjmu.edu.cn
Background: Cigarette smoking has shown to be associated with sleep disturbance, especially prolonged sleep onset latency (SOL). Cigarette smoking stimulates the release of dopamine (DA) and serotonin (5-HT), which might promote awakening and inhibit rapid eye movement sleep. Dopamine transporter (DAT) and serotonin transporter play a key role in the reuptake of DA and 5-HT from the synaptic cleft into presynaptic neurons. However, the relationship among cigarette smoking, sleep disturbance and neurotransmitters has never been investigated in human cerebrospinal fluid (CSF).

Methods: A total of 159 Chinese male subjects ( 81 active smokers and 78 non-smokers) who would undergo lumbar puncture before the surgery of anterior cruciate ligament reconstruction were recruited and 5mL-CSF samples were collected incidentally. CSF levels of DA, DAT, 5-HT, and serotonin transporter were measured using radioimmunoassay and ELISA. Sociodemographic data and the Pittsburgh Sleep Quality Index (PSQI) scale were collected before surgery.

Results: PSQI global scores, SOL, and CSF DA levels were significantly higher in active smokers compared to non-smokers $(2.00[1.00-4.75]$ scores vs $4.00[3.00-6.00]$ scores, $p=0.001 ; 10.00$ [5.00-15.00] minutes vs $15.00[10.00-30.00]$ minutes, $p=0.002 ; 87.20[82.31-96.06] \mathrm{ng} / \mathrm{mL}$ vs 107.45 [92.78-114.38] ng/mL, $p<0.001$ ), while CSF DAT levels were significantly lower in active smokers $(0.35[0.31-0.39] \mathrm{ng} / \mathrm{mL}$ vs $0.29[0.26-0.34] \mathrm{ng} / \mathrm{mL}, p<0.001)$.

Conclusion: Cigarette smoking was indeed associated with sleep disturbance, shown by prolonged SOL, higher DA levels and lower DAT levels in CSF of active smokers.

Keywords: sleep, cigarette smoking, dopamine, cerebrospinal fluid

\section{Introduction}

Cigarette smoking has been shown to be associated with insomnia. Insomnia is the most common sleep disorder prevalent in $10 \%$ to $15 \%$ of the general population characterized by difficulty in either initiating sleep or maintaining sleep continuity, and poor sleep quality. ${ }^{1}$ A population based study exhibited a relationship between cigarette smoking, difficulty in initiating sleep, and symptoms associated with sleep fragmentation, and active smokers were significantly more likely to report difficulty in sleeping. ${ }^{2}$ Also, other studies showed similar results that active smokers frequently report difficulty in initiating sleep, ${ }^{3,4}$ namely prolonged sleep onset latency (SOL).

Sleep is proven to vacillate between two distinct stages of the NREM and REM stages. Dopamine (DA) and serotonin (5-HT) are showed to promote awakening ${ }^{5,6}$ and inhibit REM sleep (REMS) ${ }^{6-8}$ suggesting their association with the transition between being 
awake and sleep, while cigarette smoking stimulates the release of $\mathrm{DA}^{9}$ and 5 -HT. ${ }^{10} \mathrm{DA}$ is a highly potent wakepromoting signal in mammals, ${ }^{11}$ and DA depletion in mice promotes the complete suppression of REMS. ${ }^{12}$ To keep the proper signaling levels, DA needs to be cleared from the synaptic cleft by the dopamine transporter (DAT). DAT has a key role in the reuptake of DA from the synaptic cleft into presynaptic dopamine neurons. ${ }^{13}$ DAT knockout mice showed higher wakefulness and less slow wave sleep. ${ }^{5}$ Cigarette smoking regulates the DA and DAT in animals and human beings' studies. Nicotine-induced DA release is significantly elevated in the nucleus accumbens of rats by microdialysis ${ }^{14}$ and in the striatum of rats by positron emission tomography. ${ }^{15}$ DA was significantly elevated in the caudate in smoker autopsies, ${ }^{16}$ while DAT availability showed a decrease in the striatum of male smokers by single photon emission computed tomography. ${ }^{17}$ The bulk of neuroanatomical data available indicates that DA-containing neurons in the brain receive a prominent innervation from 5-HT originating in the raphe nuclei of the brainstem. ${ }^{18}$ DA neuronal cell bodies and terminal sites are modulated to interact with the 5-HT system of brain regions. ${ }^{19} 5$-HT and its transporter play roles in the modulation of sleep, ${ }^{20}$ and maintain wakefulness. ${ }^{21} 5$-HT receptor knockout mice show a significant increase of wakefulness which has been partly ascribed to the increase of dopaminergic neurotransmission. ${ }^{8}$ Extracellular levels of 5-HT in the brain seem to follow the firing pattern of the raphe neurons, being highest during the wake, lower during slow wave sleep and lowest during REM sleep. ${ }^{22}$ Serotonin transporter (SERT) plays a role similar to DAT. SERT knockout mice exhibit more REM sleep than wild type litter mates and display more frequent REM sleep bouts that last longer. ${ }^{23}$ Moreover, chronic high-dose nicotine exposure has been reported to result in agespecific decreases in adolescent SERT densities. ${ }^{24}$

The cerebrospinal fluid (CSF) which is considered as a better reflection of the biochemical characteristics in the brain might be the preferable sample to study the central nervous system. Therefore, this present study was aimed to apply CSF samples to investigate the association among cigarette smoking, levels of DA, 5-HT or their transporters, and sleep disturbance.

\section{Participants and Methods Study Design}

In this cross-sectional study, active smokers were treated as cases, and non-smokers were matched by sex and age as controls.

\section{Participants}

Due to extremely few female smokers in China, ${ }^{25}$ we consecutively recruited 159 Han Chinese male subjects who had scheduled for surgery of anterior cruciate ligament reconstruction from 2014 to 2016, including 81 active smokers and 78 non-smokers. Sociodemographic data, including age and years of education, and clinical data such as the history of substance abuse and dependence were obtained according to self-report and confirmed by the next of kin and family members. The exclusion criteria included (1) systemic or central nervous system diseases or (2) a family history of psychosis and neurological diseases, determined by the MiniInternational Neuropsychiatric Interview.

Participants without a history of any substance abuse or dependence including cigarette smoke, alcohol, opioids, marijuana, et al, were assigned to the non-smoker group. Active smokers were defined as those who consumed half a pack of cigarettes (half pack $=10$ cigarettes) per day at least for one year, and had no other psychiatric disorders according to the Diagnostic and Statistical Manual of Mental Disorders, 4th Edition. Considering sleep disturbance correlated with increased smoking urge, ${ }^{26}$ active smokers were then grouped into moderate smokers (smoking $\geq 10$ and $<20$ cigarettes per day for at least 1 year) and heavy smokers (smoking $\geq 20$ cigarettes per day for at least 1 year) based on the number of cigarettes smoked per day based on the standard of WHO criteria.

The present study was approved by the Inner Mongolian Medical University Institutional Review Board and conducted in accordance with the Declaration of Helsinki and obtained written informed consent from all subjects.

\section{Assessments}

The information on demographic and clinical characteristics was obtained from all subjects and their medical records. The data of high-density lipoprotein (HDL), lowdensity lipoprotein (LDL), alanine aminotransferase test (ALT), cholesterol (CHO), triglyceride (TG), gammaglutamyl transferase (GGT) and aspartate aminotransferase (AST) came from routine tests of the subjects to evaluate physical condition on admission. These peripheral metabolic marker levels were measured in the morning on the second hospital day after an overnight fasting period using a biochemistry analyzer (HITACH 7600, Hitachi Co., Tokyo, Japan). The smoking related 
variables were collected from active smokers: age at smoking onset, years of cigarette smoking, average daily amount of cigarette smoking, and maximum daily amount of cigarettes smoking. Those questionnaires, including SOL, were all finished in 24 hours before anterior cruciate ligament reconstructive surgery by all subjects. As well, the Self-Rating Anxiety Scale (SAS), ${ }^{27}$ 13-item Beck Depression Inventory (BDI), ${ }^{28}$ and Chinese version of Pittsburgh Sleep Quality Index (PSQI) scale, ${ }^{29}$ were completed in three days before the surgery by all subjects to evaluate the anxious and depressive condition and sleep quality.

The SAS consists of 20 items to detect anxiety in research and clinical practice, and the total score may vary from 20 (no anxiety at all) to 80 (severe anxiety). The 13-item BDI is a self-completion questionnaire that is widely used to assess the severity of depressive symptoms with each item rated from 0 to 3 to indicate the least-tomost depressed mood. Its score ranges of cutoff points for various categories of depression are 0-4 (No Depression), 5-7 (Mild), 8-15 (Moderate), and 16-39 (Severe). The PSQI measures sleep quality retrospectively over the previous month and consists of 19 items combined to seven components, including subjective sleep quality, sleep latency, sleep duration, habitual sleep efficiency, sleep disturbance, sleep medication use, and daytime dysfunction. Each component score can range from 0 to 3 . The seven component scores are summed to yield a PSQI global score ranging from 0 to 21 , with a cutoff of 8 in Chinese people, ${ }^{30,31}$ such that the higher score indicates poorer sleep quality.

\section{Biological Sample Collections and Laboratory Tests}

Lumbar puncture which is part of the standard clinical practice for patients undergoing anterior cruciate ligament reconstructive surgery in China was performed in the morning before surgery by a licensed anesthetist and a $5 \mathrm{~mL}$ CSF sample was obtained via intrathecal collection divided into tubes and immediately frozen at $-80^{\circ} \mathrm{C}$ for storage. The time interval between assessments and lumbar puncture was less than 24 hours.

Analyses were performed to measure CSF levels of 5-HT (15-2500 ng/mL) and DA (4.5-2000 ng/mL) using radioimmunoassay kits (LDN, Nordhorn, Germany), and DAT (0.156-10 ng/mL) and SERT (0.156-10 ng/mL) using ELISA kits (Cloud-clone Corp., Katy, TX, USA), respectively. The CSF samples were diluted with buffer in the volume ratio of 1:9 for SERT. Laboratory technicians were blinded to clinical data.

\section{Statistical Analysis}

Since the data of this study did not follow a normal distribution, the Wilcoxon rank sum tests and one-way analysis of variance (ANOVA) were used to compare the differences of general demographic, clinical data and raw biomarkers between groups. Analysis of covariance (ANCOVA) for continuous variables was used to examine the differences between groups. Pearson correlation and partial correlation analysis were performed to determine the correlation between continuous variables and smoking related variables, adjusted for age and years of education, respectively. Bonferroni adjustment was also conducted to deal with multiple comparisons. All statistical analyses were performed using IBM SPSS Statistics for Windows, Version 20.0 (IBM Corp., Armonk, NY, USA) with a significance level of 0.05 and two-sided test.

\section{Results}

\section{Demographic and Clinical Characteristics}

The longer education years were observed in non-smokers compared to active smokers (15.00 [11.00-15.00] years vs 11.00 [8.00-15.00] years, $p=0.001$ ), while no significant difference was found in age, SAS and BDI scores, and clinical characteristics between two groups (all $p>0.05$ ). The SAS and BDI score of all subjects were $\leq 45$ scores and $\leq 13$ scores, respectively. Both the PSQI global scores and SOL were significantly higher in active smokers compared to non-smokers $(2.00[1.00-4.75]$ scores vs 4.00 [3.00-6.00] scores, $p=0.001 ; 10.00$ [5.00-15.00] minutes vs 15.00 [10.00-30.00] minutes, $p=0.002$, respectively, shown in Table 1).

\section{CSF Biomarkers Analysis}

Using ANCOVA adjusted for years of education and all four biomarkers as covariates, both the PSQI global scores and SOL were higher in active smokers compared to nonsmokers ( $p=0.003$ and $p=0.002$, respectively). The CSF DAT levels were found significantly lower in active smokers, while the CSF DA levels were significantly higher in active smokers (both $p<0.001$ ), shown in Table 2. The CSF SERT levels were significantly higher in active smokers $(p=0.031)$, but there was no significance after the Bonferroni correction. 
Table I Clinical Characteristics of Non-Smokers and Active Smokers

\begin{tabular}{|c|c|c|c|}
\hline Variables & Non-Smokers $(n=78)$ & Active Smokers $(n=8 I)$ & $p$ \\
\hline Age (years) & $31.00(26.75-40.25)$ & $32.00(27.00-42.00)$ & 0.403 \\
\hline Education (years) & $15.00(11.00-15.00)$ & II.00 (8.00-15.00) & $0.001 *$ \\
\hline $\mathrm{SAS}^{\#}$ & $35.00(33.00-36.00)$ & $35.00(31.25-37.00)$ & 0.582 \\
\hline $\mathrm{BDI}^{\#}$ & $1.00(0-2.00)$ & $2.00(0.50-3.00)$ & 0.057 \\
\hline PSQI global scores"\# & $2.00(1.00-4.75)$ & $4.00(3.00-6.00)$ & $0.00 I^{*}$ \\
\hline Sleep onset latency ${ }^{\#}$ (minute) & $10.00(5.00-15.00)$ & $15.00(10.00-30.00)$ & $0.002^{*}$ \\
\hline $\mathrm{HDL}(\mathrm{mM} / \mathrm{L})$ & $1.23(1.09-1.35)$ & $1.19(1.06-1.35)$ & 0.464 \\
\hline LDL (mM/L) & $2.77(2.35-3.12)$ & $2.63(2.27-2.96)$ & 0.245 \\
\hline ALT (U/L) & $26.00(17.75-37.75)$ & $26.00(14.50-42.50)$ & 0.688 \\
\hline $\mathrm{CHO}(\mathrm{mM} / \mathrm{L})$ & $4.85(4.29-5.27)$ & $4.71(4.36-5.28)$ & 0.662 \\
\hline $\mathrm{TG}(\mathrm{mM} / \mathrm{L})$ & $1.74(1.27-2.44)$ & $1.51(1.06-1.90)$ & 0.042 \\
\hline GGT (U/L) & $32.50(23.75-48.75)$ & $32.00(23.00-59.00)$ & 0.957 \\
\hline AST (U/L) & $21.00(17.00-24.00)$ & $20.00(15.00-25.00)$ & 0.339 \\
\hline CSF Dopamine (ng/mL) & $87.20(82.31-96.06)$ & $107.45(92.78-1 \mid 4.38)$ & $<0.001 *$ \\
\hline CSF DAT (ng/mL) & $0.35(0.31-0.39)$ & $0.29(0.26-0.34)$ & $<0.001 *$ \\
\hline CSF 5-HT (ng/mL) & $302.14(284.05-326.76)$ & $285.20(274.05-297.50)$ & $<0.001 *$ \\
\hline CSF SERT $(\mathrm{ng} / \mathrm{mL})$ & 83.29 (73.45-90.42) & 83.29 (76.36-92.49) & 0.306 \\
\hline
\end{tabular}

Notes: All data were reported as median (IQR) using the Mann-Whitney U-test; "Using ANOVA; *p $<0.05$.

Abbreviations: SAS, Self-Rating Anxiety Scale; BDI, Beck Depression Inventory; PSQI, Pittsburgh Sleep Quality Index; HDL, high-density lipoprotein; LDL, low-density lipoprotein; ALT, alanine aminotransferase; CHO, cholesterol; TG, triglyceride; GGT, gamma-glutamyl transferase; AST, aspartate aminotransferase; CSF, cerebral spinal fluid; DAT, dopamine transporter; 5-HT, serotonin; SERT, serotonin transporter; IQR, interquartile range; ANOVA, one-way analysis of variance.

Table 2 CSF Biomarkers Differences Between Non-Smokers and Active-Smokers

\begin{tabular}{|l|l|l|l|}
\hline & Mean Differences & $95 \%$ Cl & P \\
\hline PSQI global scores & -1.87 & $-3.08,-0.66$ & $0.003^{*}$ \\
Sleep onset latency (minute) & -10.81 & $-17.43,-4.19$ & $0.002^{*}$ \\
CSF Dopamine $(\mathrm{ng} / \mathrm{mL})$ & -16.42 & $-26.61,-11.24$ & $<0.00 I^{*}$ \\
CSF DAT $(\mathrm{ng} / \mathrm{mL})$ & 0.06 & $0.03,0.08$ & $<0.00 I^{*}$ \\
CSF 5-HT $(\mathrm{ng} / \mathrm{mL})$ & 11.23 & $-3.83,26.27$ & 0.142 \\
CSF SERT $(\mathrm{ng} / \mathrm{mL})$ & -7.92 & $-15.10,-0.74$ & $0.03 I^{*}$ \\
\hline
\end{tabular}

Notes: ANCOVA was used for PSQI global scores and sleep onset latency with years of education and four biomarkers as covariates; ANCOVA was used for each biomarker with years of education and other biomarkers as covariates; $*_{p}<0.05$.

Abbreviations: PSQI, Pittsburgh Sleep Quality Index; CSF, cerebral spinal fluid; DAT, dopamine transporter; 5-HT, serotonin; SERT, serotonin transporter; Cl, confidence interval; ANCOVA, analysis of covariance.

\section{Correlation and Difference in Active Smokers}

Using partial correlation adjusted for age, years of education, scores of SAS and BDI, and smoking related variables, there was no correlation of the PSQI global scores and SOL with four CSF biomarkers (all $p>0.05$ ). There was no correlation of the PSQI global scores and SOL with smoking related variables using partial correlation adjusted for age, years of education, scores of SAS and BDI, and the other smoking related variables (all $p>0.05$ ).

A total of 81 active smokers were grouped into moderate smokers $(n=47)$ and heavy smokers $(n=34)$. No difference was found in age, years of education, BDI scores, PSQI global scores, SOL, and other clinical characteristics between two groups, except for $\mathrm{CHO}$ (4.86 [4.56-5.40] $\mathrm{mM} / \mathrm{L}$ vs 4.47[4.07-4.99] $\mathrm{mM} / \mathrm{L}, p=0.005)$ and SAS scores $(34.50[30.00-36.00]$ scores vs 36.00 [34.00-38.00] scores, $p=0.021$ ) (shown in Table 3). There was no significant difference in CSF levels of DA, DAT, 5-HT and SERT between moderate and heavy smokers, adjusted for age, years of education and other biomarkers.

\section{Discussion}

This is the first study in human CSF to investigate the association among cigarette smoking, neurotransmitters 
Table 3 Clinical Characteristics of Moderate Smokers and Heavy Smokers

\begin{tabular}{|c|c|c|c|}
\hline Variables & Moderate Smokers $(n=47)$ & Heavy Smokers $(n=34)$ & $p$ \\
\hline Age (years) & $32.00(25.00-43.00)$ & $33.00(28.50-42.00)$ & 0.716 \\
\hline Education (years) & $11.00(11.00-15.00)$ & $11.00(8.00-15.00)$ & 0.235 \\
\hline SAS $^{\#}$ & $34.50(30.00-36.00)$ & $36.00(34.00-38.00)$ & $0.021 *$ \\
\hline $\mathrm{BDI}^{\#}$ & $2.00(1.00-4.00)$ & $2.00(0-2.00)$ & 0.233 \\
\hline PSQI global scores" & $3.00(2.00-6.00)$ & $4.00(3.00-6.00)$ & 0.865 \\
\hline Sleep onset latency ${ }^{\#}$ (minute) & $20.00(10.00-30.00)$ & $15.00(5.00-27.50)$ & 0.254 \\
\hline $\mathrm{HDL}(\mathrm{mM} / \mathrm{L})$ & $1.23(1.11-1.42)$ & $1.15(0.97-1.31)$ & 0.070 \\
\hline LDL (mM/L) & $2.63(2.38-2.92)$ & $2.49(2.08-3.01)$ & 0.215 \\
\hline ALT (U/L) & $24.00(14.00-42.00)$ & $30.50(15.50-44.25)$ & 0.500 \\
\hline $\mathrm{CHO}(\mathrm{mM} / \mathrm{L})$ & $4.86(4.56-5.40)$ & $4.47(4.07-4.99)$ & $0.005^{*}$ \\
\hline TG (mM/L) & $1.59(1.08-2.21)$ & $1.36(1.03-1.57)$ & 0.122 \\
\hline GGT (U/L) & $31.50(22.75-57.50)$ & $32.00(24.50-69.00)$ & $0.44 I$ \\
\hline AST (U/L) & $19.00(16.00-24.00)$ & $21.00(13.75-25.25)$ & 0.886 \\
\hline CSF Dopamine (ng/mL) & $107.90(95.28-118.30)$ & $102.60(91.70-111.20)$ & 0.065 \\
\hline CSF DAT (ng/mL) & $0.29(0.26-0.34)$ & $0.29(0.25-0.35)$ & 0.736 \\
\hline CSF 5-HT (ng/mL) & $286.40(274.45-297.50)$ & $283.75(270.95-298.53)$ & 0.376 \\
\hline CSF SERT $(\mathrm{ng} / \mathrm{mL})$ & $80.62(76.36-92.49)$ & $83.79(74.50-90.68)$ & $0.78 \mathrm{I}$ \\
\hline
\end{tabular}

Notes: All data were reported as median (IQR) using the Mann-Whitney U-test; ${ }^{*}$ Using ANOVA; ${ }^{*} p<0.05$.

Abbreviations: SAS, Self-Rating Anxiety Scale; BDI, Beck Depression Inventory; PSQI, Pittsburgh Sleep Quality Index; HDL, high-density lipoprotein; LDL, low-density lipoprotein; ALT, alanine aminotransferase; CHO, cholesterol; TG, triglyceride; GGT, gamma-glutamyl transferase; AST, aspartate aminotransferase; CSF, cerebral spinal fluid; DAT, dopamine transporter; 5-HT, serotonin; SERT, serotonin transporter; IQR, interquartile range; ANOVA, one-way analysis of variance.

with sleep disturbance. The primary finding showed that cigarette smoking was indeed associated with sleep disturbance, shown by poorer sleep quality, prolonged SOL, as well as higher DA levels and lower DAT levels in CSF of active smokers compared to non-smokers. Previous studies have demonstrated that cigarette smoking is associated with exacerbated sleep quality in adults and adolescents using polysomnography. ${ }^{32-34}$ Studies on large cohorts of smokers have shown a reduction of subjectively sleep quality and more insomnia-like symptoms, including poor sleep quality, long time to fall asleep and less restorative sleep. ${ }^{35,36}$ Several polysomnographic studies reported that active smokers have a longer SOL and a higher percentage of time in the NREM $\mathrm{N} 1$ stage resulting in shallower and poor sleep significantly compared to nonsmokers. ${ }^{33,37}$ Also, better sleep quality is associated with withdrawal. ${ }^{26}$ By positron emission tomography, a human study showed that cigarette smoking induced the upregulation of DA levels in the limbic striatum which correlates with the urge to smoke. Elevations in extracellular DA induced by smoking have been associated to the craving or improved mood, which can continue to promote smoking behavior and further increase the DA release. ${ }^{38}$ Nicotine as the primary psychoactive ingredient in tobacco promotes the DA release. $9,39-41$ Nicotinic acetylcholine receptors are expressed presynaptically on dopamine axon terminals, and their chronic desensitization reduces the dynamic range of dopamine release in response to dopamine cell activity and contrast between high and low dopamine activity. ${ }^{42}$ This might enhance the urge to smoke to promote DA release in another way.

The role of DA as a sleep suppressor has been well recognized, and induced arousal states are associated with altered DA neurotransmission. ${ }^{43}$ DA is involved in increased wakefulness in animal experiments and human studies. On the contrary, the depletion of DA in humans provokes sleepiness. $^{12}$ The DAT transports DA against a concentration gradient from the synapse into the intracellular space as the primary mechanism and provides spatial and temporal control of the dopamine signal. ${ }^{44}$ Recently, a series of molecular imaging studies showed a significant reduction in DAT availability and function in active smokers compared to non-smokers. ${ }^{17,45,46}$ DA uptake by the DAT is electrogenic (bringing negative charge into the cell) and abolishing the internal positive charge at the plasma membrane by depolarization would counteract rather than promote DA uptake. Consequently, membrane trafficking was responsible for DAT downregulation, ${ }^{47}$ which explained the DAT decrease in active smokers of our study.

In addition, nicotine induces an increase of 5-HT levels in some brain regions. ${ }^{48}$ However, nicotine activates an increase of DA in merely a few seconds, ${ }^{49}$ and produces minimal effects on 5-HT neurons ${ }^{50}$ with the induced release 
of 5-HT much lower. ${ }^{51}$ Also, 5-HT and DA are metabolic degraded by monoamine oxidase, and 5-HT neurons have greater regulation by monoamine oxidase than DA. ${ }^{51}$ So, though a lower level of 5-HT was not found in active smokers, the decrease of 5-HT could depend on a greater activation of monoamine oxidase. Besides, in our study, no correlation was observed between sleep variables and DA or 5-HT, which might result from that PSQI reflecting the overall sleep quality could not reflect the length of REM sleep. DA and 5HT are recognized to be correlated with length of REM sleep in normal conditions, the extra stimulation of nicotine affecting the secretion and function of DA and 5HT might be another reason. The laboratory tests of blood levels of HDL, LDL, ALT, CHO, TG, GGT and AST were only a part of the routine examination on admission. The previous studies showed that cigarette smoking could damage the metabolic function of liver, ${ }^{52,53}$ which might explain the difference of $\mathrm{CHO}$ levels between the groups.

There are some limitations to the present study. First, CSF cannot directly reflect neuron changes during sleep; nevertheless, it is considered to represent biochemical changes in the brain, unlike DA and 5-HT plasma levels; DA, 5-HT and many other substances cannot cross the blood brain barrier. ${ }^{54-56}$ Secondly, participants recruited in this study were subjects with anterior cruciate ligament injuries instead of healthy populations, which might be seen as a confounder when interpreting the results. Finally, cigarette smoking has also effects on other neurotransmitters, and we will make deep research in this field in the future.

\section{Conclusion}

Cigarette smoking was indeed associated with sleep disturbance, shown by prolonged SOL, higher DA levels and lower DAT levels in CSF of active smokers.

\section{Abbreviations}

SOL, sleep onset latency; DA, dopamine; 5-HT, serotonin; REM, rapid eye movement; NREM, non-rapid eye movement; DAT, dopamine transporter; SERT, serotonin transporter; CSF, cerebral spinal fluid; PSQI, Pittsburgh Sleep Quality Index; SAS, Self-Rating Anxiety Scale; BDI, Beck Depression Inventory; ANOVA, one-way analysis of variance; ANCOVA, analysis of covariance; HDL, highdensity lipoprotein; LDL, low-density lipoprotein; ALT, alanine aminotransferase; $\mathrm{CHO}$, total cholesterol; TG, triglyceride; GGT, gamma-glutamyl transferase; AST, aspartate aminotransferase.

\section{Acknowledgments}

The authors thank all of the participants for their willingness to participate in the study and the time that they devoted to the study. The authors thank Drs Srinivasan Nithiyanantham and Hui-Chih Chang for English language polishing.

\section{Funding}

This work was supported by the following grants: The Technology Support Project of Xinjiang (2017E0267), Natural Science Foundation of Xinjiang Province (2018D01C228 and 2018D01C239), Tianshan Youth Project-Outstanding Youth Science and Technology Talents of Xinjiang (2017Q007), Natural Science Foundation of China (81421004), the 10th Inner Mongolia Grassland Talents Project, Beijing Natural Science Foundation (7152074), and The Opening Project of Zhejiang Provincial Top Key Discipline of Pharmaceutical Sciences.

\section{Disclosure}

The authors report no conflicts of interest for this work.

\section{References}

1. Buysse DJ. Chronic insomnia. Am J Psychiatry. 2008;165 (6):678-686. doi:10.1176/appi.ajp.2008.08010129

2. Jefferson CD, Drake CL, Scofield HM, et al. Sleep hygiene practices in a population-based sample of insomniacs. Sleep. 2005;28 (5):611-615. doi:10.1093/sleep/28.5.611

3. Wetter DW, Young TB. The relation between cigarette smoking and sleep disturbance. Prev Med. 1994;23(3):328-334. doi:10.1006/ pmed.1994.1046

4. Brook DW, Rubenstone E, Zhang C, Brook JS. Trajectories of cigarette smoking in adulthood predict insomnia among women in late mid-life. Sleep Med. 2012;13(9):1130-1137. doi:10.1016/j. sleep.2012.05.008

5. Wisor JP, Nishino S, Sora I, Uhl GH, Mignot E, Edgar DM. Dopaminergic role in stimulant-induced wakefulness. J Neurosci. 2001;21(5):1787-1794. doi:10.1523/JNEUROSCI.21-05-01787.2001

6. Monti JM. The role of dorsal raphe nucleus serotonergic and non-serotonergic neurons, and of their receptors, in regulating waking and rapid eye movement (REM) sleep. Sleep Med Rev. 2010;14 (5):319-327. doi:10.1016/j.smrv.2009.10.003

7. Monti JM, Monti D. The involvement of dopamine in the modulation of sleep and waking. Sleep Med Rev. 2007;11(2):113-133. doi:10.1016/j.smrv.2006.08.003

8. Monti JM. Serotonin control of sleep-wake behavior. Sleep Med Rev. 2011;15(4):269-281. doi:10.1016/j.smrv.2010.11.003

9. Sesack SR, Grace AA. Cortico-Basal Ganglia reward network: microcircuitry. Neuropsychopharmacology. 2010;35(1):27-47.

10. Lowery CL, Elliott C, Cooper A, et al. Cigarette smoking-associated alterations in serotonin/adrenalin signaling pathways of platelets. J Am Heart Assoc. 2017;6(5). doi:10.1161/JAHA.116.005465

11. Andretic R, van Swinderen B, Greenspan RJ. Dopaminergic modulation of arousal in Drosophila. Curr Biol. 2005;15(13):1165-1175. doi:10.1016/j.cub.2005.05.025

12. Dzirasa K, Ribeiro S, Costa R, et al. Dopaminergic control of sleep-wake states. $J$ Neurosci. 2006;26(41):10577-10589. doi:10.1523/JNEUROSCI.1767-06.2006 
13. Ueno T, Kume K. Functional characterization of dopamine transporter in vivo using Drosophila melanogaster behavioral assays. Front Behav Neurosci. 2014;8:303. doi:10.3389/fnbeh.2014.00303

14. Dewey SL, Brodie JD, Gerasimov M, Horan B, Gardner EL, Ashby CR. A pharmacologic strategy for the treatment of nicotine addiction. Synapse. 1999;31(1):76-86. doi:10.1002/(SICI)10982396(199901)31:1<76::AID-SYN10>3.0.CO;2-Y

15. Brody AL, Olmstead RE, London ED, et al. Smoking-induced ventral striatum dopamine release. Am J Psychiatry. 2004;161(7):1211-1218.

16. Court JA, Lloyd S, Thomas N, et al. Dopamine and nicotinic receptor binding and the levels of dopamine and homovanillic acid in human brain related to tobacco use. Neuroscience. 1998;87(1):63-78. doi:10.1016/S0306-4522(98)00088-8

17. Yang YK, Yao WJ, Yeh TL, et al. Decreased dopamine transporter availability in male smokers - a dual isotope SPECT study. Prog Neuropsychopharmacol Biol Psychiatry. 2008;32(1):274-279. doi:10.1016/j.pnpbp.2007.08.018

18. Di Giovanni G, Di Matteo V, Pierucci M, Esposito E. Serotonindopamine interaction: electrophysiological evidence. Prog Brain Res. 2008; 172:45-71.

19. Kapur S, Remington G. Serotonin-dopamine interaction and its relevance to schizophrenia. Am J Psychiatry. 1996;153(4):466-476.

20. Oikonomou G, Altermatt M, Zhang RW, et al. The serotonergic raphe promote sleep in zebrafish and mice. Neuron. 2019;103(4):686701e688. doi:10.1016/j.neuron.2019.05.038

21. Ursin R. Serotonin and sleep. Sleep Med Rev. 2002;6(1):55-69. doi:10.1053/smrv.2001.0174

22. Portas CM, Bjorvatn B, Ursin R. Serotonin and the sleep/wake cycle: special emphasis on microdialysis studies. Prog Neurobiol. 2000;60 (1):13-35. doi:10.1016/S0301-0082(98)00097-5

23. Wisor JP, Wurts SW, Hall FS, et al. Altered rapid eye movement sleep timing in serotonin transporter knockout mice. Neuroreport. 2003;14(2):233-238. doi:10.1097/00001756-200302100-00015

24. Collins SL, Wade D, Ledon J, Izenwasser S. Neurochemical alterations produced by daily nicotine exposure in periadolescent vs. adult male rats. Eur J Pharmacol. 2004;502(1-2):75-85. doi:10.1016/j. ejphar.2004.08.039

25. Ma Y, Wen L, Cui W, et al. Prevalence of cigarette smoking and nicotine dependence in men and women residing in two provinces in China. Front Psychiatry. 2017;8:254. doi:10.3389/fpsyt.2017.00254

26. Purani H, Friedrichsen S, Allen AM. Sleep quality in cigarette smokers: associations with smoking-related outcomes and exercise. Addict Behav. 2019;90:71-76. doi:10.1016/j.addbeh.2018.10.023

27. Zung WW. A rating instrument for anxiety disorders. Psychosomatics. 1971;12(6):371-379.

28. Beck AT, Rial WY, Rickels K. Short form of depression inventory: cross-validation. Psychol Rep. 1974;34(3):1184-1186.

29. Buysse DJ, Reynolds CF, Monk TH, Berman SR, Kupfer DJ. The Pittsburgh sleep quality index: a new instrument for psychiatric practice and research. Psychiatry Res. 1989;28(2):193-213. doi:10.1016/0165-1781(89)90047-4

30. Guo S, Sun W, Liu C, Wu S. Structural validity of the Pittsburgh sleep quality index in Chinese undergraduate students. Front Psychol. 2016;7:1126.

31. Qi DG, Liu R, Wu X-X, Pang J, Deng J, Wang A. Investigation on sleeping quality of university students and its influential factors. Mod Prev Med. 2007;5.

32. McNamara JP, Wang J, Holiday DB, et al. Sleep disturbances associated with cigarette smoking. Psychol Health Med. 2014;19 (4):410-419. doi:10.1080/13548506.2013.832782

33. Jaehne A, Unbehaun T, Feige B, Lutz UC, Batra A, Riemann D. How smoking affects sleep: a polysomnographical analysis. Sleep Med. 2012;13(10):1286-1292. doi:10.1016/j.sleep.2012.06.026

34. Dugas EN, Sylvestre MP, O’Loughlin EK, et al. Nicotine dependence and sleep quality in young adults. Addict Behav. 2017;65:154-160. doi:10.1016/j.addbeh.2016.10.020
35. Riedel BW, Durrence HH, Lichstein KL, Taylor DJ, Bush AJ. The relation between smoking and sleep: the influence of smoking level, health, and psychological variables. Behav Sleep Med. 2004;2 (1):63-78. doi:10.1207/s15402010bsm0201 6

36. Sabanayagam C, Shankar A. The association between active smoking, smokeless tobacco, second-hand smoke exposure and insufficient sleep. Sleep Med. 2011;12(1):7-11. doi:10.1016/j.sleep.2010.09.002

37. Patterson F, Grandner MA, Malone SK, Rizzo A, Davey A, Edwards DG. Sleep as a target for optimized response to smoking cessation treatment. Nicotine Tob Res. 2019;21(2):139-148. doi:10.1093/ntr/ntx236

38. Le Foll B, Guranda M, Wilson AA, et al. Elevation of dopamine induced by cigarette smoking: novel insights from a [11C]-+-PHNO PET study in humans. Neuropsychopharmacology. 2014;39 (2):415-424. doi:10.1038/npp.2013.209

39. Liang K, Poytress BS, Chen Y, Leslie FM, Weinberger NM, Metherate R. Neonatal nicotine exposure impairs nicotinic enhancement of central auditory processing and auditory learning in adult rats. Eur J Neurosci. 2006;24(3):857-866. doi:10.1111/j.1460-9568.2006.04945.x

40. Koob GF, Volkow ND. Neurocircuitry of addiction. Neuropsychopharmacology. 2010;35(1):217-238.

41. Schilstrom B, Nomikos GG, Nisell M, Hertel P, Svensson TH. $\mathrm{N}$-methyl-D-aspartate receptor antagonism in the ventral tegmental area diminishes the systemic nicotine-induced dopamine release in the nucleus accumbens. Neuroscience. 1998;82(3):781-789. doi:10.1016/S0306-4522(97)00243-1

42. Koranda JL, Cone JJ, McGehee DS, Roitman MF, Beeler JA, Zhuang X. Nicotinic receptors regulate the dynamic range of dopamine release in vivo. $J$ Neurophysiol. 2014;111(1):103-111. doi:10.1152/jn.00269.2013

43. Kume K, Kume S, Park SK, Hirsh J, Jackson FR. Dopamine is a regulator of arousal in the fruit fly. $J$ Neurosci. 2005;25 (32):7377-7384. doi:10.1523/JNEUROSCI.2048-05.2005

44. Cragg SJ, Rice ME. DAncing past the DAT at a DA synapse. Trends Neurosci. 2004;27(5):270-277. doi:10.1016/j.tins.2004.03.011

45. Ashok AH, Mizuno Y, Howes OD. Tobacco smoking and dopaminergic function in humans: a meta-analysis of molecular imaging studies. Psychopharmacology. 2019;236(4):1119-1129. doi:10.1007/ s00213-019-05196-1

46. Danielson K, Putt F, Truman P, Kivell BM. The effects of nicotine and tobacco particulate matter on dopamine uptake in the rat brain. Synapse. 2014;68(2):45-60. doi:10.1002/syn.21715

47. Melikian HE, Buckley KM. Membrane trafficking regulates the activity of the human dopamine transporter. J Neurosci. 1999;19 (18):7699-7710. doi:10.1523/JNEUROSCI.19-18-07699.1999

48. Seth P, Cheeta S, Tucci S, File SE. Nicotinic-serotonergic interactions in brain and behaviour. Pharmacol Biochem Behav. 2002;71 (4):795-805. doi:10.1016/S0091-3057(01)00715-8

49. Rossi S, Singer S, Shearman E, Sershen H, Lajtha A. The effects of cholinergic and dopaminergic antagonists on nicotine-induced cerebral neurotransmitter changes. Neurochem Res. 2005;30(4):541-558. doi:10.1007/s11064-005-2689-x

50. Wei C, Han X, Weng D, et al. Response dynamics of midbrain dopamine neurons and serotonin neurons to heroin, nicotine, cocaine, and MDMA. Cell Discov. 2018;4:60. doi:10.1038/s41421-018-0060-z

51. Hashemi P, Dankoski EC, Lama R, Wood KM, Takmakov P, Wightman RM. Brain dopamine and serotonin differ in regulation and its consequences. Proc Natl Acad Sci U S A. 2012;109 (29):11510-11515. doi:10.1073/pnas.1201547109

52. Craig WY, Palomaki GE, Haddow JE. Cigarette smoking and serum lipid and lipoprotein concentrations: an analysis of published data. BMJ. 1989;298(6676):784-788. doi:10.1136/bmj.298.6676.784

53. Kuzuya M, Ando F, Iguchi A, Shimokata H. Effect of smoking habit on age-related changes in serum lipids: a cross-sectional and longitudinal analysis in a large Japanese cohort. Atherosclerosis. 2006;185(1):183-190. doi:10.1016/j.atherosclerosis.2005.05.040 
54. Koller WC, Rueda MG. Mechanism of action of dopaminergic agents in Parkinson's disease. Neurology. 1998;50(6Suppl 6):S11-14;discussion S44-18.

55. Berger M, Gray JA, Roth BL. The expanded biology of serotonin. Annu Rev Med. 2009;60:355-366. doi:10.1146/annurev.med.60.042307.11 0802
56. Sumara G, Sumara O, Kim JK, Karsenty G. Gut-derived serotonin is a multifunctional determinant to fasting adaptation. Cell Metab. 2012;16(5):588-600. doi:10.1016/j.cmet.2012.09.014

\section{Publish your work in this journal}

Nature and Science of Sleep is an international, peer-reviewed, open access journal covering all aspects of sleep science and sleep medicine, including the neurophysiology and functions of sleep, the genetics of sleep, sleep and society, biological rhythms, dreaming, sleep disorders and therapy, and strategies to optimize healthy sleep.
The manuscript management system is completely online and includes a very quick and fair peer-review system, which is all easy to use. Visit http://www.dovepress.com/testimonials.php to read real quotes from published authors. 